\title{
Adsorption and Separation of Aromatic Amino Acids from Aqueous Solutions Using Metal-Organic Frameworks
}

\author{
Dries Jonckheere, ${ }^{\dagger}$ Julian A. Steele, ${ }^{\dagger}$ B Birgit Claes, $^{\dagger}{ }^{\dagger}$ Bart Bueken, ${ }^{\dagger}$ Laurens Claes, ${ }^{\dagger}$ Bert Lagrain, ${ }^{\dagger}$
} Maarten B. J. Roeffaers, ${ }^{\dagger}$ and Dirk E. De Vos ${ }^{* \dagger}$

${ }^{\dagger}$ Department of Microbial and Molecular Systems, Centre for Surface Chemistry and Catalysis (COK), Leuven Chem\&Tech, KU Leuven, Celestijnenlaan 200F, Postbox 2461, 3001 Leuven, Belgium

\section{Supporting Information}

ABSTRACT: Metal-organic frameworks (MOFs) are investigated for the adsorption of aromatic amino acids L-phenylalanine (L-Phe), Ltryptophan (L-Trp), and L-tyrosine (L-Tyr) from aqueous solutions. After screening a range of water-stable MOFs, the hydrophobic $\mathrm{Zr}$ MOF MIL-140C emerged as the best performing material, exhibiting uptakes of 15 wt \% for L-Trp and 20 wt \% for L-Phe. These uptakes are 5-10 wt \% higher than those of large-pore zeolites Beta and Y. Both single-compound and competitive adsorption isotherms for L-Phe and $\mathrm{L}$-Trp were experimentally obtained at the natural $\mathrm{pH}$ of these amino acid mixtures ( $\mathrm{pH}$ 6.5-7) without additional $\mathrm{pH}$ modification. We find that the hydrophobic nature of MIL-140C and the capacity of LTrp to form hydrogen bonds favor the uptake of L-Trp with its larger indole moiety compared to the smaller phenyl side group of L-Phe. On the basis of literature and vibrational analysis, observations of hydrogen-bonded L-Trp within the MIL-140C framework are evidenced by red- and blue-shifted -NH vibrations (3400 $\mathrm{cm}^{-1}$ ) in Fourier transform infrared spectroscopy, which were attributed to types $\mathrm{N}-\mathrm{H}_{\mathrm{L}-\mathrm{Trp}} \cdots \pi_{\mathrm{MIL}-140 \mathrm{C}}$ and $\mathrm{N}-\mathrm{H}_{\mathrm{L}-\mathrm{Trp}} \cdots \mathrm{O}_{\mathrm{MIL}-140 \mathrm{C}}$, respectively. MIL-140C is shown to be recycled at least three times for both aromatic amino acids without any loss of adsorption capacity, separation performance, or crystallinity. Desorption of aromatic amino acids proceeds easily in aqueous ethanol. Substantial coadsorption of negatively charged amino acids L-glutamate and L-aspartate (L-Glu and L-Asp) was observed from a model solution for wheat straw protein hydrolysate at $\mathrm{pH} 4.3$. On the basis of these results, we conclude that MIL-140C is an interesting material for the recovery of essential aromatic amino acids L-Tyr, L-Phe, and L-Trp and of L-Glu and L-Asp from waste protein hydrolysates.

KEYWORDS: metal-organic framework, MOF, adsorption, separation, aromatic amino acid, amino acids, tryptophan, phenylalanine, protein waste

\section{INTRODUCTION}

The aromatic amino acids (AAA) L-phenylalanine (L-Phe), Ltyrosine (L-Tyr), and L-tryptophan (L-Trp) are categorized as essential amino acids and require uptake through the diet. ${ }^{1}$ These compounds are key intermediates in metabolism, for instance in the biosynthesis of neurotransmitters, hormones, and vitamins. ${ }^{2,3}$ Their applications are mainly situated in food, feed and pharmaceutical industries: L-Phe is widely applied as a precursor for the artificial sweetener aspartame together with Laspartic acid (L-Asp), while L-Tyr and L-Trp are important nutritional supplements. Next to applications in the food industry, other amino acids such as L-glutamic acid (L-Glu) and L-lysine (L-Lys) are considered as interesting biobased platform molecules. ${ }^{4-6}$

The amino acid market is rapidly expanding ( 6 billion USD at 3 Mtons/year in 2007) $)^{7}$ and is expected to reach 20 to 30 billion USD by $2020 .{ }^{8,9}$ Although aromatic amino acids are produced on a much smaller scale $(0.5 \%$ or 16000 tons/year in 2007) than L-Glu or L-Lys, they still contribute to about $5 \%$ of the yearly amino acid revenue. This share is expected to increase.

Amino acids are synthesized industrially via fermentation ${ }^{10,11}$ or chemoenzymatic processes, e.g. L-Phe by enantioselective addition of ammonia to trans-cinnamic acid. ${ }^{4}$ Alternatively, amino acids can also be recovered by hydrolyzing nonfeed protein waste. Examples of such waste include poultry feather meal and vinasses of sugar cane and sugar beet, which are readily available from agroindustry and bioethanol production. ${ }^{4,12}$ Averaged mass fractions of aromatic amino acids in such protein-rich waste stream hydrolysates vary between 5 and $10 \mathrm{wt} \% .^{12}$ Selective separation of amino acids from aqueous media is a key step in each of these processes, but the development of high-performance separation techniques remains challenging.

Received: June 26, 2017

Accepted: August 7, 2017

Published: August 7, 2017 
Table 1. Single-Compound Uptake of AAAs L-Phe and L-Trp on MOFs, Zeolites, Mesoporous Silica, and Ion-Exchange Resins Based on Literature Data and Experimental Work

\begin{tabular}{|c|c|c|c|c|c|c|c|}
\hline \multirow[b]{2}{*}{ entry } & \multirow[b]{2}{*}{ material } & \multicolumn{2}{|c|}{ AAA uptake (wt \%) } & \multirow[b]{2}{*}{ remark } & \multirow[b]{2}{*}{$\mathrm{pH}$} & \multicolumn{2}{|c|}{$[\mathrm{AAA}]_{\text {initial }}(\mathrm{M})$} \\
\hline & & L-Phe & L-Trp & & & L-Phe & L-Trp \\
\hline 1 & $\mathrm{CuCHDC}$ & 0 & 0 & MOF breakdown & 6.5 & 0.05 & 0.05 \\
\hline 2 & ZIF-8 & 0 & 0 & MOF breakdown & 6.5 & 0.05 & 0.05 \\
\hline 3 & $\mathrm{UiO}-66$ & 9.9 & 4.1 & & 6.5 & 0.05 & 0.05 \\
\hline 4 & MIL-140B & 3.6 & 3.3 & & 6.5 & 0.05 & 0.05 \\
\hline 5 & MIL-140C & 19.7 & 15.4 & & 6.5 & 0.15 & 0.05 \\
\hline 6 & MIL-140D & 8.4 & 8.5 & & 6.5 & 0.05 & 0.05 \\
\hline 7 & MIL-68(Al)-TFA & 14.9 & 21.9 & & 6.5 & 0.05 & 0.05 \\
\hline 8 & MOF-808 & 55.1 & 32.4 & & 6.5 & 0.15 & 0.05 \\
\hline 9 & ZSM-5 $\left(\mathrm{H}^{+}\right)^{23}$ & 4.8 & nd & $\mathrm{Si} / \mathrm{Al}=30$ & 6 & nd & \\
\hline 10 & ZSM-5 $\left(\mathrm{Na}^{+}\right)^{24}$ & 4.3 & 0 & $\mathrm{Si} / \mathrm{Al}=80$ & 6 & 0.6 & nd \\
\hline 11 & ZSM-5 $\left(\mathrm{H}^{+}\right)^{\text {this work }}$ & 0.81 & 0 & $\mathrm{Si} / \mathrm{Al}=140$ & 6.5 & 0.05 & 0.05 \\
\hline 12 & Beta $\left(\mathrm{H}^{+}\right)^{26}$ & 6.6 & nd & $\mathrm{Si} / \mathrm{Al}=12$ & 9 & 0.05 & \\
\hline 13 & Beta $\left(\mathrm{H}^{+}\right)^{23}$ & 13.9 & nd & $\mathrm{Si} / \mathrm{Al}=25$ & 6 & nd & \\
\hline 14 & Beta $\left(\mathrm{H}^{+}\right)^{25}$ & 7.4 & nd & $\mathrm{Si} / \mathrm{Al}=48$ & 4.5 & 0.06 & \\
\hline 15 & $\mathrm{Y}\left(\mathrm{Na}^{+} / \mathrm{H}^{+}\right)^{26}$ & 0.8 & nd & $\mathrm{Si} / \mathrm{Al}=2.5$ & 6.6 & 0.03 & \\
\hline 16 & CBV-400 $\left[\mathrm{Y}\left(\mathrm{H}^{+}\right)\right]^{\text {this work }}$ & 5.8 & 4.9 & $\mathrm{Si} / \mathrm{Al}=2.5$ & 6.5 & 0.05 & 0.05 \\
\hline 17 & CBV-100 $\left[\mathrm{Y}\left(\mathrm{Na}^{+}\right)\right]^{\text {this work }}$ & $<1$ & $<1$ & $\mathrm{Si} / \mathrm{Al}=2.5$ & 6.5 & 0.05 & 0.05 \\
\hline 18 & $\mathrm{Y}\left(\mathrm{Na}^{+}\right)^{26}$ & 16.5 & nd & $\mathrm{Si} / \mathrm{Al}=8$ & 6.4 & 0.11 & \\
\hline 19 & SBA- $15^{31}$ & 6.6 & nd & & 5.5 & 0.035 & \\
\hline 20 & $\mathrm{D} 008\left(\mathrm{H}^{+}\right)^{19}$ & nd & 61.6 & macroporous, strong & $3-5$ & & 0.025 \\
\hline 21 & $\mathrm{HZ}-001\left(\mathrm{H}^{+}\right)^{19}$ & nd & 75.4 & gel type, strong & $3-5$ & & 0.025 \\
\hline 22 & DOWEX $50 \mathrm{Wx} 2\left(\mathrm{H}^{+}\right)$ & 70.3 & 74.9 & gel-type, strong & 6.5 & 0.15 & 0.05 \\
\hline 23 & DOWEX MAC3 $\left(\mathrm{H}^{+}\right)$ & 7.8 & 10.5 & macroporous, weak & 6.5 & 0.05 & 0.05 \\
\hline
\end{tabular}

Recovery techniques such as crystallization, electrodialysis, ${ }^{13}$ reactive extraction, ${ }^{14}$ and combined techniques such as flotation-complexation-extraction, ${ }^{15}$ nanofiltration over polyelectrolyte membranes, ${ }^{16}$ and adsorption using ionic liquids ${ }^{17}$ have already been explored. Often, such methods are prone to high energy consumption or are impractical to implement. Thus, the adsorption of amino acids on solid porous materials is a worthwhile alternative. For instance, ion-exchange resins are able to separate amino acids based on their $\mathrm{pH}$-dependent charge in water. ${ }^{18-21}$ When aromatic ion-exchange resins are used, $\mathrm{pH}$ modification of the aqueous medium can facilitate uptake by adding electrostatic interactions to the strong dispersive forces $(\pi-\pi)$ : the $\mathrm{pH}$ is modified so that either $\mathrm{pH}>\mathrm{pK}$ a $-\mathrm{NH}_{3}{ }^{+}(\approx 9.2)$ for anion-exchangers or so that $\mathrm{pH}$ $<\mathrm{p} K_{\mathrm{a}}$ of $-\mathrm{COOH}(\approx 2.2)$ for cation-exchangers. These materials are also known to swell considerably in water, which involves a large volume expansion of the resin by intercalation of water molecules between the resin's polymer chains. This phenomenon ensures the accessibility of nearly all ion-exchange sites and allows additional $\pi-\pi$ interactions of adsorbing aromatic amino acids with the resin's aromatic backbone, resulting in high adsorption uptakes. However, regeneration of these adsorbents remains an important drawback because highly acidic or alkaline conditions are required to disrupt the strong electrostatic adsorbentadsorbate interactions, ultimately producing amino acid salts. Inorganic materials such as zeolites ${ }^{22-29}$ and mesoporous molecular sieves ${ }^{30-32}$ display a somewhat lower amino acid uptake due to their lower ion-exchange capacity and a lack of swelling in comparison to ion-exchange resins.

Metal-organic frameworks (MOFs) are a relatively new (but already well-established) class of materials consisting of metalion nodes and multitopic organic linkers which assemble into microporous frameworks. ${ }^{33-35}$ They are endowed with high surface areas and are highly tailorable, which makes them attractive for applications in chemistry and environmental science. MOFs have been intensely researched in a wide variety of fields, including gas separation and purification, ${ }^{36}$ catalysis, $^{37,38}$ luminescence, ${ }^{39-41}$ drug delivery, ${ }^{42}$ and liquid-phase separations, ${ }^{43}$ among many others.

Few studies involved amino acids in MOF chemistry to date: amino acids have been used as (i) modulator or nucleationinducing reagent in the synthesis of respectively $\mathrm{UiO}-66^{44,45}$ and ZIF-8, ${ }^{46}$ or (ii) for postsynthetic linker functionalization with L-proline (L-Pro) to yield a catalytically active $\mathrm{Cu}-\mathrm{MOF}{ }^{47}$ Only the group of Kitagawa looked into amino acid adsorption on MOFs, reporting L-Glu adsorption on isoreticular Al-based MOFs with loadings from 7 to $15 \mathrm{wt} \% .{ }^{48}$ Full desorption was accomplished in a phosphate buffer while maintaining the crystallinity of these Al-based MOFs.

In this paper, the adsorption behavior of AAAs on several water-stable MOFs is investigated. The hydrophobic and waterstable Zr-4,4'-biphenyldicarboxylate MIL-140C (Matériaux de $1^{\prime}$ Institut Lavoisier) emerges as the most promising material. It is further evaluated for batch and dynamic adsorption of both single-compound and binary amino acid mixtures in both batch and continuous operation. Additionally, the adsorption mechanism for L-Trp was studied by Fourier transform infrared spectroscopy (FT-IR) and vibrational analysis, revealing that hydrogen bonds play an important role in its relatively high uptake. Finally, the adsorption behavior of MIL-140C in complex amino acid mixtures with up to 17 amino acids was probed. 


\section{RESULTS AND DISCUSSION}

Materials Screening. Several water-stable MOFs were probed for the adsorption of aromatic amino acids. The results were compared to literature data and to our own experimental data for zeolites and ion-exchange resins, as summarized in Table 1. Full experimental details are given in the Supporting Information. In short, the amino acids were dissolved at room temperature in Milli- $Q$ water with a concentration range between 0 and $0.05 \mathrm{M}$ for L-Trp and $0.15 \mathrm{M}$ for L-Phe in singlecompound adsorption tests. For competitive adsorption, both AAAs are present in the same concentration $(0-0.05 \mathrm{M}$ for both). The $\mathrm{pH}$ of the solution was not further modified. The AAA uptakes were calculated from HPLC (high pressure liquid chromatography) UV detector output data by comparing the initial concentration of the AAA solution to the concentrations after exposure to the different tested adsorbents (MOFs, zeolites, and ion-exchange resins).

The adsorption of aromatic amino acids was evaluated for some well-known water-stable MOFs (Table 1, entries 1-8) at $0.05 \mathrm{M}$ for L-Phe and $0.05 \mathrm{M}$ for L-Trp. The tested materials are CuCHDC, ZIF-8, UiO-66, MIL-140B-C-D, MIL-68(Al)-TFA, and MOF-808. Although ZIF-8 and CuCHDC (Table 1, entries 1 and 2) have been reported to be stable in the separation of bioalcohols ${ }^{49}$ or butanol isomers ${ }^{50}$ from aqueous fermentation media, these frameworks were degraded after contact with the amino acids $(2 \mathrm{~h})$ as a result of the chelating properties of these molecules. Powder X-ray diffraction (PXRD) measurements indeed showed structural transformation to insoluble $\mathrm{M}^{2+}$. phenylalaninato ${ }^{51}$ and $\mathrm{M}^{2+}$-tryptophanato ${ }^{52,53}$ complexes (Figures S1A and B) reported for $\mathrm{Cu}^{2+}$ and $\mathrm{Ni}^{2+}$ or $\mathrm{Mn}^{2+}$, respectively. This result is remarkable, as amino acids have been proven to act as biomimetic crystallization agents for ZIF-8 in aqueous synthesis solutions. ${ }^{46}$ However, the latter observation was made at amino acid concentrations much lower compared to the concentrations of the present adsorption work. Therefore, other MOF materials with hard, higher valent metal cations $\left(\mathrm{Al}^{3+}, \mathrm{Zr}^{4+}\right)$ were tested, as their $\mathrm{M}^{x+}$-carboxylate bonds are less prone to hydrolysis. ${ }^{54}$ Indeed, these materials retain their structure after soaking in aqueous solutions of AAAs, and adsorption thereof was observed (see Figures S1C$\mathrm{G}$ and Table 1, entries 3-8). The Zr-terephthalate UiO-66 (Table 1, entry 3 ) already achieves uptakes of $10 \mathrm{wt} \%$ for L-Phe and 4 wt \% for L-Trp, similar to values reported for large-pore zeolites.

Adsorption of aromatic amino acids on MIL-140(Zr)-type materials (Table 1 entries 4-6) follows similar trends in optimal pore size and intrapore polarity, as described previously for phenolics adsorption. ${ }^{54}$ In this family of materials, the linker size and hence pore size is systematically increased in the series: MIL-140B (2,6-naphthalenedicarboxylate), MIL-140C (4,4'biphenyldicarboxylate), and MIL-140D (3,3'-dichloro-4,4' -azobenzenedicarboxylate). However, the adsorption capacity decreases in the order MIL-140C > MIL-140D > MIL-140B. This reflects that in the very large and more polar pores of MIL-140D few specific interactions exist with the pore walls, while in the smaller pores of MIL-140B there are too many spatial constraints to achieve substantial uptake capacity. The material MIL-140C strikes an optimal balance with a maximal adsorption capacity of $20 \mathrm{wt} \%$ for L-Phe and $15 \mathrm{wt} \%$ for L-Trp.

Alternatively, we evaluated MOFs with a high density of open coordination sites or missing-linker defects after thermal treatment, such as MIL-68(Al)-TFA and MOF-808 (Table 1, entries 7-8). These materials are usually synthesized in relatively high concentrations of the modulating agents trifluoroacetic acid (TFA) and formic acid, respectively. A high uptake of L-Phe (55 wt \%) and L-Trp (32 wt \%) in MOF808 can be explained by the large pore volume of this framework. However, the full adsorption capacity does not yet seem to be reached at maximum solubility of the respective amino acids (i.e., no plateau is reached in the adsorption isotherm), and the adsorption isotherms have a weak initial slope (Figure S1). Another explanation is that the high density of open coordination sites in these frameworks leads to higher polarity of the frameworks and competitive adsorption of water molecules. These factors point toward a fairly low affinity of these two large-pore materials for AAAs. Therefore, despite their high uptake, MIL-68-TFA(Al) and MOF-808 are not investigated further.

Previously, AAA adsorption was studied extensively on other porous frameworks. In particular, L-Phe adsorption on zeolites was studied in terms of $\mathrm{pH}$ effects and $\mathrm{Si} / \mathrm{Al}$ ratios. In general, the $\mathrm{pH}$ of the amino acid mixture is of little concern as long as the amino acid occurs predominantly in the zwitterionic form, i.e. in the $\mathrm{pH}$ range between 2.2 and 9.2. ${ }^{23} \mathrm{ZSM}-5$, a hydrophobic zeolite with medium pore size $(\approx 5.8 \AA$ ) , disfavors the uptake of L-Trp compared to that of L-Phe and L-Tyr based on their kinetic diameters of $6.5,5.8$, and $5.9 \AA$, respectively (Table 1, entries 9-11). ${ }^{24}$ Large-pore zeolites such as the faujasite Y zeolite and zeolite Beta display remarkably higher uptakes of L-Phe than ZSM-5 (Table 1, entries 12-18; up to 16 wt \%). For these materials, the $\mathrm{Si} / \mathrm{Al}$ ratio needs tuning to achieve significant adsorption. On the one hand, zeolites with low $\mathrm{Si} / \mathrm{Al}$ ratios contain many cation-exchange sites, which makes them very hydrophilic: the uptake of AAAs with their aromatic rings is reduced due to competitive adsorption of water. On the other hand, zeolites with high $\mathrm{Si} / \mathrm{Al}$ ratios contain only a limited number of cation-exchange sites, and their frameworks may become too apolar to enable amino acid adsorption in the case that these molecules occur as zwitterions. These trends are very well-illustrated in the literature (Table 1, entries 12-14, 15, and 16) and are confirmed by some of our own observations (Table 1, entries 11, 16, and 17). Additionally, mesoporous materials such as all-silica SBA-15 (Table 1, entry 19) show considerable L-Phe adsorption of $6 \mathrm{wt}$ $\%$ at low concentrations.

In contrast to zeolites, ion-exchange resins show much higher uptake of (aromatic) amino acids for various reasons (Table 1 , entries 20-23): these materials (i) contain strong ion-exchange sites, (ii) have additional adsorbent-adsorbate interactions due to $\pi-\pi$ stacking, and (iii) are able to swell. Polystyrene/ divinylbenzene (PS/DVB)-based cation-exchange resins reported in literature with strong sulfonic acid groups reach high uptakes of 60-75 wt \% for L-Trp (Table 1, entries 20 and 21). In this work, similar results were obtained for L-Phe on DOWEX 50Wx2 (Figure S2 and Table 1, entry 24). A remarkably lower uptake was observed for a polyacrylic-based cation-exchanger with carboxylic acid functional groups such as DOWEX MAC-3 (Table 1, entry 25) because of its lower acid strength.

Batch Single-Compound Adsorption Experiments on MIL-140C. On the basis of the materials screening, MIL-140C proved to be the most promising MOF, combining high adsorption capacity for both L-Phe and L-Trp with high affinity for these aromatic amino acids. The single-compound adsorption isotherms for L-Phe and L-Trp on MIL-140C 
(Figure 1) display typical type-1 adsorption behavior with steep initial slopes, especially for L-Trp.

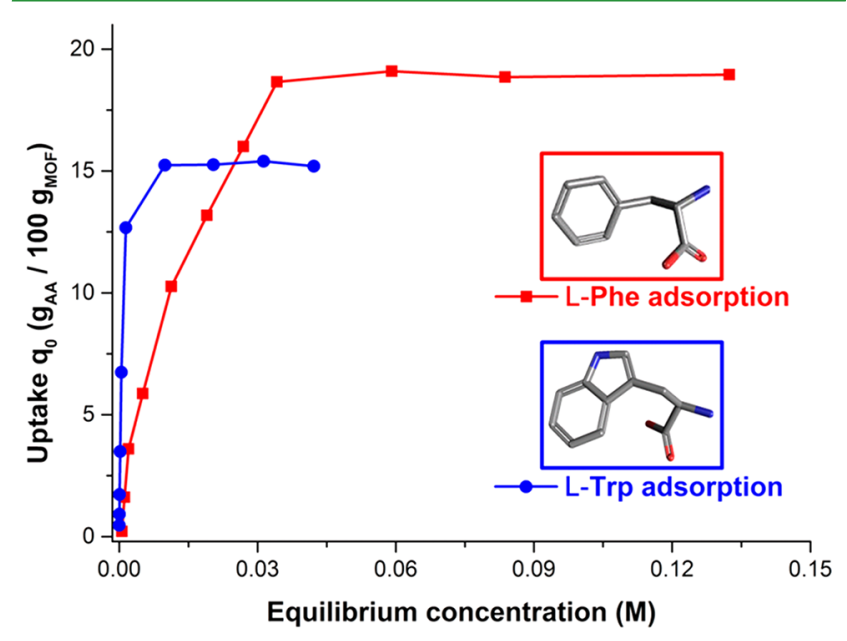

Figure 1. Single-compound adsorption isotherms for L-Phe ([LPhe $\left.]_{\max }=0.15 \mathrm{M}\right)$ and L-Trp $\left([\mathrm{L}-\mathrm{Trp}]_{\max }=0.05 \mathrm{M}\right)$ on MIL-140C.

The PXRD patterns of pristine MIL-140C and after adsorption of L-Phe and L-Trp show no crystallinity loss (Figure 2). The relative intensity shifts of the first two reflections and the appearance of an additional reflection at $11.4^{\circ} 2 \theta$ are indicative of pore-filling in the MIL-140C framework. $^{54}$



Figure 2. PXRD patterns of MIL-140C before and after L-Phe and LTrp adsorption.

MIL-140C can be recycled at least three times for the adsorption and desorption of L-Phe and L-Trp without any loss of capacity or crystallinity (Figures 3 and S3). Amino acid desorption from MIL-140C was achieved in aqueous ethanol (4:1 v/v $\left.\mathrm{H}_{2} \mathrm{O}: \mathrm{EtOH}\right)$, as proven by FT-IR after washing (Figure S4). Compared to the desorption of molecules from ion-exchange resins with strong acids or bases, this mild desorption from MIL-140C with aqueous ethanol is far more sustainable and economical. Moreover, the amino acids are desorbed as pure zwitterionic compounds from MIL-140C using aqueous ethanol rather than as salts, which is the case when they are desorbed from ion-exchange resins with acids or bases.

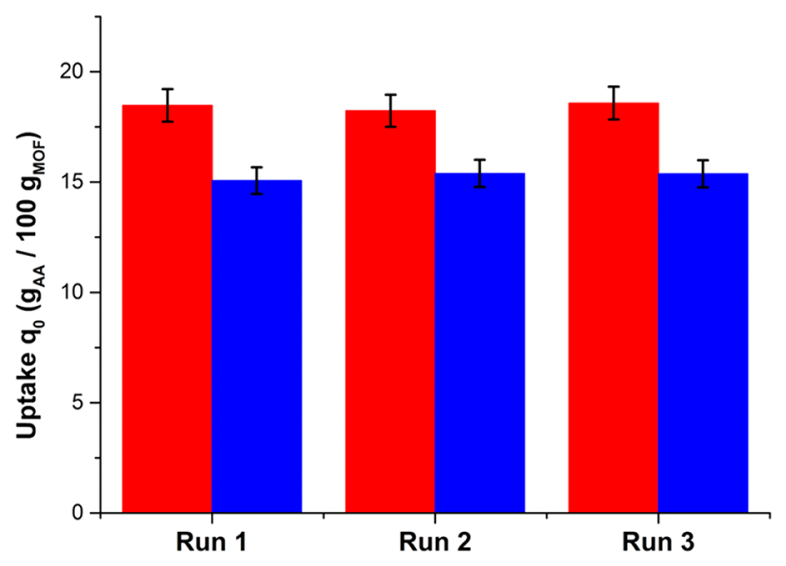

Figure 3. Recycling of MIL-140C for the single-compound adsorption of L-Phe (red) and L-Trp (blue) for three consecutive runs.

Elucidating L-Trp Preference over L-Tyr and L-Phe on MIL-140C. The initial slopes of the adsorption isotherms in Figure 4 clearly reveal an affinity of MIL-140C for L-Trp that is

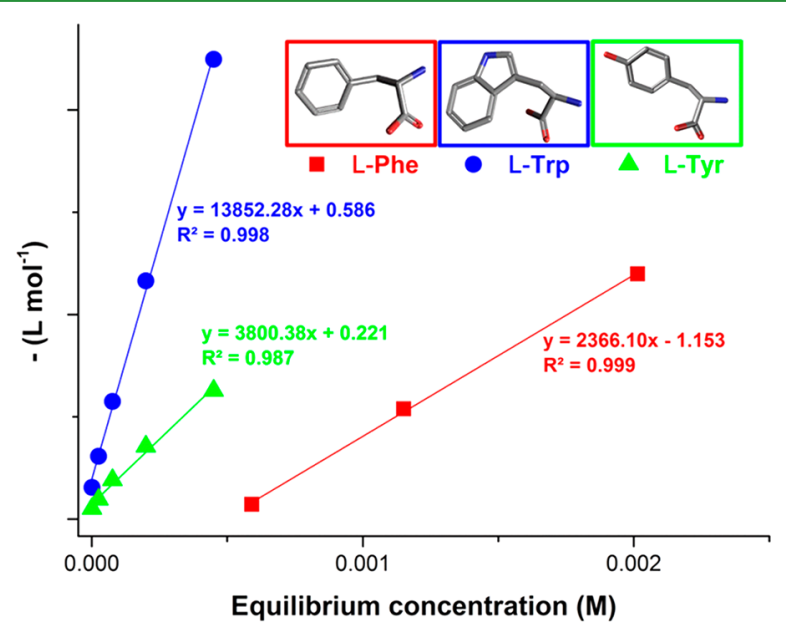

Figure 4. Initial slopes of single-compound adsorption isotherms of LPhe, L-Trp, and L-Tyr on MIL-140C. The steeper the initial slope, the higher the affinity of the adsorbent for the adsorbate.

respectively three to four times higher than that for L-Phe and L-Tyr. As expected for a hydrophobic material such as MIL$140 \mathrm{C}$, the largest heterocycle (L-Trp) was adsorbed with the highest affinity compared to the smaller molecule L-Phe. However, it is peculiar that the hydrophobic framework MIL$140 \mathrm{C}$ has an affinity for more polar molecules L-Tyr (and LTrp) higher than that for the less polar L-Phe (Figure 4). From previous work, it is known that compounds with phenolic hydroxyl groups strongly interact with the carboxylate group in the double $\mathrm{Zr}$ oxide chain (consisting of $\mathrm{ZrO}_{7}$ polyhedra) of MIL-140C. Similar interactions are plausible for L-Tyr and presumably also for L-Trp. ${ }^{54}$ However, a full adsorption isotherm for L-Tyr could not be determined due to its low solubility in water at the natural $\mathrm{pH}$ of 6.8 (2.4 mM; Figure S5).

To directly confirm the presence of adsorbed amino acids within the MIL-140C framework and gain insights into the nature of the adsorption mechanism at the molecular level, FTIR spectroscopy was employed. Figure 5A displays FT-IR spectra recorded for MIL-140C, L-Phe, and L-Trp adsorbed on MIL-140C along with the spectra of the pure amino acids for comparison. The main emphasis of this FT-IR study lies in the 


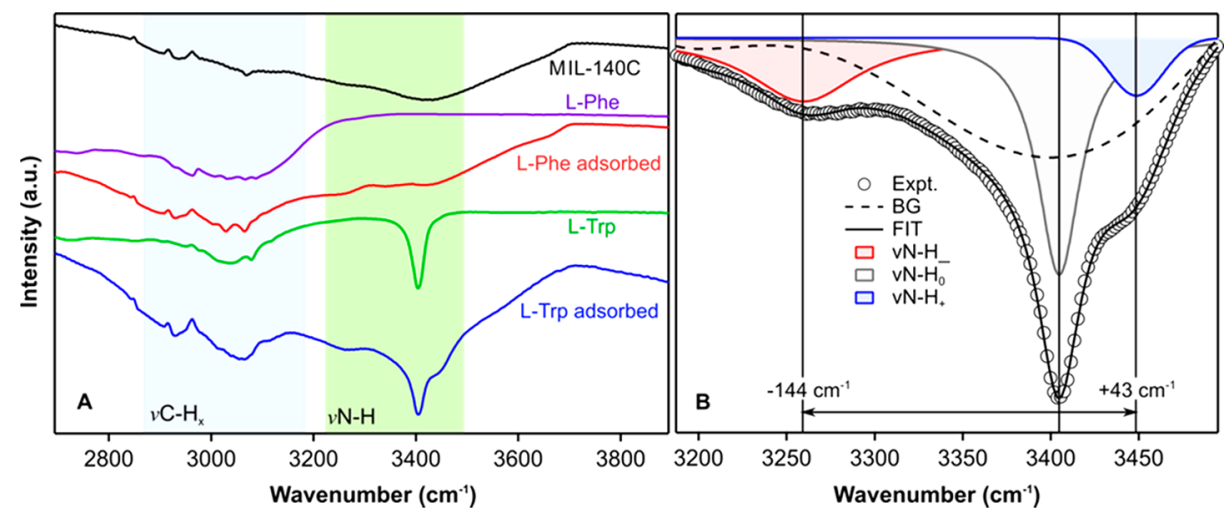

Figure 5. (A) FT-IR spectra of MIL-140C, L-Phe, and L-Trp along with their adsorbed versions. For clarity, these spectra have been rescaled and offset with the $\nu \mathrm{C}-\mathrm{H}_{x}$ and $\nu \mathrm{N}-\mathrm{H}$ vibrational energy bands identified. (B) Resolved spectrum of L-Trp adsorbed on MIL-140C expanded over the $\mathrm{N}-\mathrm{H}$ stretching frequency range with the background (BG) and various vibrational contributions identified.

high-energy $\mathrm{C}-\mathrm{H}$ and $\mathrm{N}-\mathrm{H}$ stretching frequency range; the full recorded spectra can be found in Figure S6A of the Supporting Information. The adsorption of L-Phe and L-Trp within MIL-140C is clear with the introduction of $\mathrm{C}-\mathrm{H}_{x}$ vibrational signatures in the vicinity of $3000 \mathrm{~cm}^{-1}$ which are not present in the parent material (only weak, narrow $\mathrm{C}-\mathrm{H}_{x} x$ $=1$ feature is seen in MIL-140C). Additionally, for the spectrum of L-Trp adsorbed on MIL-140C, a sharp absorption band of the indole secondary amine $(\mathrm{N}-\mathrm{H})$ centered near $3400 \mathrm{~cm}^{-1}$ is observed. For completeness, the weaker lowenergy modes of adsorbed L-Phe and L-Trp are further identified in Figure S6B of the Supporting Information. As mentioned above, a likely explanation for the enhanced uptake of L-Trp in MIL-140C compared to L-Phe resides in the highly polar and versatile nature of the $\mathrm{N}-\mathrm{H}$ bond in the indole moiety and its capacity to establish hydrogen bonds. ${ }^{55}$ Such an adsorption interaction is known to induce vibrational changes to the interacting groups relative to the unperturbed vibrational state. In general, establishing a H-bond can introduce a red(bond length elongation) or blue-shift (bond length shortening) in the mode energy. Furthermore, they will also respectively fall into the weak or strong interaction energy regimes. ${ }^{56}$ Unlike a blue-shifting $\mathrm{H}$-bond, a red-shifting one experiences no upper limit to its interaction energy, permitting relatively large frequency shifts to manifest. ${ }^{56}$

Within this context, the FT-IR spectrum of L-Trp adsorbed on MIL-140C is replotted over the $\nu \mathrm{N}-\mathrm{H}$ frequency range in Figure 5B for detailed treatment. Here, an agreeable fit is made by summing three Lorentzian oscillators (unperturbed $\nu \mathrm{N}-\mathrm{H}_{0}$, red-shifted $\nu \mathrm{N}-\mathrm{H}_{-}$and blue-shifted $\left.\nu \mathrm{N}-\mathrm{H}_{+}\right)$and the background trace $(\mathrm{BG})$, which is the rescaled result of fitting the broad $\mathrm{H}_{2} \mathrm{O}$ feature in the MIL-140C spectrum (see Figure 5A) with a fifth order polynomial over the same spectral range. In contrast to the single sharp absorption feature at $3400 \mathrm{~cm}^{-1}$ observed in pure L-Trp (due to $\nu \mathrm{N}-\mathrm{H}$ of the indole moiety; see Figure 5A), L-Trp adsorbed on MIL-140C distinctly exhibits a triple mode behavior with well-resolved weaker features red- $\left(\nu \mathrm{N}-\mathrm{H}_{-}\right)$and blue-shifted $\left(\nu \mathrm{N}-\mathrm{H}_{+}\right)$relative to the unperturbed peak $\left(\nu \mathrm{N}-\mathrm{H}_{0}\right)$. This observed splitting in the $\nu \mathrm{N}-\mathrm{H}$ vibrations of L-Trp adsorbed in MIL-140C confirms this functional group to be strongly interacting with the framework by forming hydrogen bonds.

Considering how L-Trp is adsorbed in MIL-140C, there are several scenarios in which $\nu \mathrm{N}-\mathrm{H}$ can shift frequency. For example, with respect to electron-rich sites across the aromatic linkers, interactions consisting of $\pi-\pi$ and $\mathrm{T}$-shaped $\mathrm{N}-\mathrm{H} \cdots \pi$ stacking, along with their rotated and tilted variations, are possible within the framework. The vibrational changes of $\nu \mathrm{N}-$ $\mathrm{H}$ experienced under such interactions have been the focus of several in-depth reports, studied both directly and indirectly using indole as an analogue. ${ }^{55,57,58}$ Consistently, all such interactions ultimately act to reduce the $\nu \mathrm{N}-\mathrm{H}$ vibrational energy. This suggests $\nu \mathrm{N}-\mathrm{H}_{-}\left(3254 \mathrm{~cm}^{-1}\right)$ in Figure $5 \mathrm{~B}$ originates from some portion of the adsorbed L-Trp being adsorbed via an interaction with the $\pi$-cloud of aromatics within the framework. In fact, a reduction of the $\nu \mathrm{N}-\mathrm{H}$ frequency is typically expected upon binding due to its large polarization. ${ }^{59}$ Therefore, resolving a blue-shifted peak near $3449 \mathrm{~cm}^{-1}(\nu \mathrm{N}-$ $\mathrm{H}_{+}$) in Figure $5 \mathrm{~B}$ is somewhat surprising, but not unprecedented for the complex type N-H... ${ }^{60-64}$ For completeness, further discussion on the phenomena of improper hydrogen bonding can be found in Supporting Note 1. Thus, we assigned $\nu \mathrm{N}-\mathrm{H}_{+}$to $\mathrm{L}$-Trp molecules improperly hydrogen bonded to oxygen atoms of MIL-140C, either linker carboxylate or oxide atoms in the $\mathrm{ZrO}_{7}$ oxide chain of MIL-140C.

A close inspection of our experimental data reveals that the full width at half-maximum (fwhm) of $\nu \mathrm{N}-\mathrm{H}_{+}\left(36 \mathrm{~cm}^{-1}\right)$ is rather narrowly distributed, relative to $\nu \mathrm{N}-\mathrm{H}_{-}\left(74 \mathrm{~cm}^{-1}\right)$, and only marginally higher than the unperturbed $\nu \mathrm{N}-\mathrm{H}$ band (30 $\mathrm{cm}^{-1}$; comparable to that measured in pure L-Trp). This may reflect the limited access for $\mathrm{L}-\mathrm{Trp}$ to establish $\mathrm{N}-\mathrm{H} \cdots \mathrm{O}$ within the framework, explaining why the interaction remains in the weak-energy regime. ${ }^{56}$ Owed to a localized shift in the dipole dynamics, hydrogen bonding is expected to impose, respectively, an increase and decrease to the absorption activity of $\nu \mathrm{N}-\mathrm{H}_{-}$and $\nu \mathrm{N}-\mathrm{H}_{+}$modes, permitting only a semiquantitative assessment of this quantity. ${ }^{56}$ The relative oscillator strengths of vibrations $\nu \mathrm{N}-\mathrm{H}_{-}$and $\nu \mathrm{N}-\mathrm{H}_{+}$are measured to be 0.68 and 0.20 , when normalized to the natural $\nu \mathrm{N}-\mathrm{H}_{0}$ mode in the same spectrum. Such spectral weighting suggests that a significant portion of $\mathrm{L}$-Trp is interacting in this manner when taken up by MIL-140C, providing additional bonding mechanisms for adsorption.

Batch Competitive Adsorption Experiments on MIL140C. After testing the single-compound adsorption behavior of MIL-140C for L-Phe and L-Trp, the separation power of this material was tested for equimolar binary mixtures. Note that based on the previous FT-IR study, additional H-bonding implies that in case of a competitive adsorption scenario, the 
incoming indole ring of L-Trp must simultaneously displace LPhe and a water molecule from its hydrogen-bonded site. The batch competitive adsorption isotherms clearly show that the uptake of L-Trp is again strongly preferred over L-Phe (Figure $6)$, as proven in the single-compound FT-IR study. The

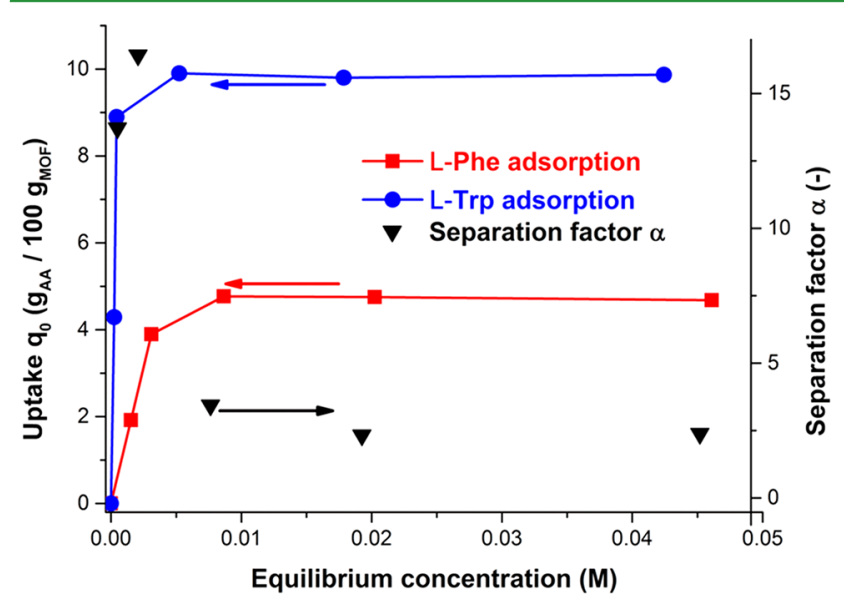

Figure 6. Competitive adsorption isotherms of L-Phe and L-Trp (0.05 M) on MIL-140C.

separation factor $\alpha$ (see Supporting Information for calculation) amounts up to a high number of 16 at low concentrations and 2 for concentrations above $0.01 \mathrm{M}$. Once more, the high affinity of MIL-140C for L-Trp is reflected by an adsorption isotherm much steeper than that for L-Phe. The maximum uptake of both compounds is independent of the temperature (Figure S7), indicating that there are no significant differences in adsorption enthalpy between L-Trp and L-Phe. The separation in MIL-140C between L-Phe and L-Trp may well be entropy-driven, as an incoming L-Trp has to displace a loosely $\pi-\pi$ stacked L-Phe and a hydrogen-bonded water molecule from the hydrogen-bond site, namely a $\mathrm{O}_{\mathrm{MIL}-140 \mathrm{C}}$ from either a linker carboxylate or the $\mathrm{Zr}$-oxide chain. This would allow L-Trp to adopt a double interaction with the framework through $\pi-\pi$ stacking and hydrogen-bonding $-\mathrm{NH}$ moiety, as verified above using vibrational analysis. This ultimately yields an entropy increase, considering the entire system (MIL-140C, L-Phe, L-Trp, and a H-bonded water molecules).

Continuous Adsorption Experiments on MIL-140C. Next, MIL-140C crystals were packed in a stainless steel column to investigate the breakthrough and adsorptive separation of L-Trp from L-Phe (both at $6.25 \mathrm{mM}$ ) under dynamic conditions $(0.5 \mathrm{~mL} / \mathrm{min})$. At this selected concentration, the uptake of both amino acids is already close to saturation capacity of MIL-140C, and the highest separation factor $\alpha$ is attained (Figure 6). The material efficiently separates both compounds, as L-Phe starts eluting about $3 \mathrm{~min}$ before LTrp (Figure 7), creating a significant window of separation. The column outlet concentration reaches the feed concentration for L-Phe after $10 \mathrm{~min}$ of operation, followed by a distinct roll-up phase, in which surplus L-Phe is displaced from MIL-140C by the more preferred L-Trp. L-Phe reaches a steady-state level after $20 \mathrm{~min}$, while a stationary level for L-Trp is reached more slowly after $40 \mathrm{~min}$ of operation. The separation factor $\alpha$ under dynamic adsorption conditions was determined to be 2.7, compared to 16 for batch mode. For L-Phe, a steep front is observed, evidencing fast diffusion in the crystals and quick establishment of the adsorption equilibrium. The lower

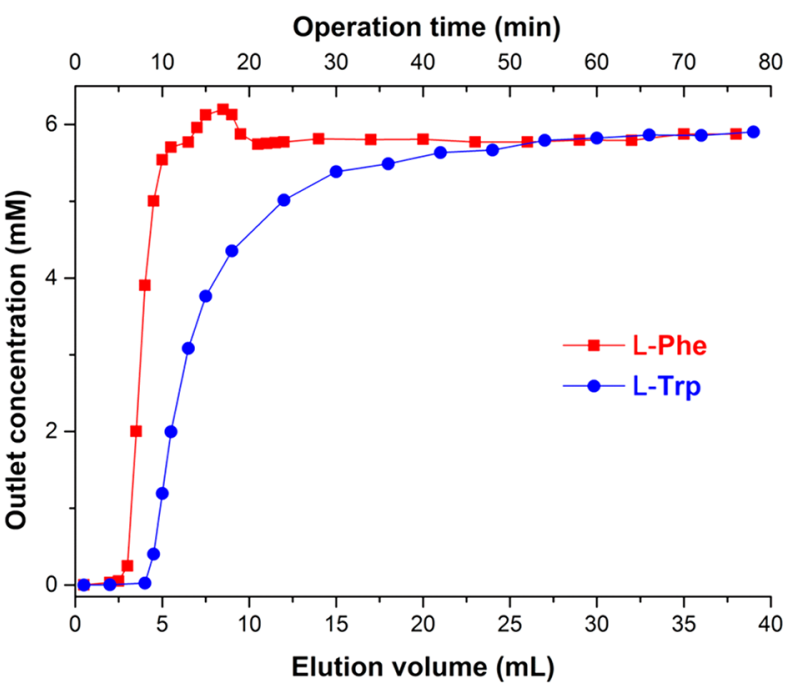

Figure 7. Breakthrough experiment of L-Phe and L-Trp (both 6.25 $\mathrm{mM}$ ) on a MIL-140C-packed column.

steepness and larger width of the front for the L-Trp may be indicative for some intraparticle diffusion limitations for this slightly larger molecule in the already well-filled monodimensional pores of MIL-140C. Similar diffusion limitations have also been observed for ion-exchange resins. ${ }^{20}$ Again, the crystallinity of the powdered MIL-140C was fully retained, even after this dynamic operation at 450 bar (Figure S8). This is in line with the known excellent mechanical stability of MIL$140 \mathrm{C}$ and related $\mathrm{Zr}-\mathrm{MOFs} .{ }^{65,66}$ Lowering the flow rate may allow to lower the operation pressure and to improve diffusion phenomena. These observations demonstrate the strong application potential of MIL-140C for aromatic amino acid adsorption in industrial multibed adsorption processes.

Amino Acid Model Mixture: Wheat Straw Protein Hydrolysate. Next, the potential of MIL-140C to enrich aromatic amino acids from complex amino acid mixtures was tested on a model mixture that mimics the amino acid composition of wheat straw protein hydrolysate, which is a nonfood agricultural waste stream. ${ }^{12}$ The commercial cationexchange resin DOWEX 50Wx2 was investigated for comparison. To investigate the influence of other amino acids on the uptake of aromatic amino acids, both materials were contacted with aqueous solutions containing (i) all amino acids, (ii) only aromatic amino acids, (iii) all amino acids except for the ones with apolar side chains (L-Val, L-Leu, L-Ile, and L-Ala), and (iv) all amino acids except for the acidic amino acids ( $\mathrm{L}-\mathrm{Glu}$ and L-Asp). Acids or bases were intentionally not added, and therefore, the adsorption experiments were performed at the natural $\mathrm{pH}$ of the amino acid mixtures. Note that L-glutamine ( $\mathrm{L}-\mathrm{Gln}$ ) and L-asparagine (L-Asn) were never included because these amino acids are converted to L-Glu and L-Asp during protein hydrolysis under acidic conditions. The initial mixture composition and an exemplary HPLC chromatogram are shown in Supporting Information, Table S2 and Figure S9, respectively. An overview of the results from these studies is contained in Table 2.

In general, the DOWEX 50Wx2 resin can adsorb a larger amount of amino acids compared to MIL-140C (Table 2). This observation parallels the trend observed during the screening and that reported in literature. Moreover, the cation-exchanging resin preferentially adsorbs positively charged, basic amino acids (L-Arg and L-Lys) in large amounts, while also 
Table 2. Uptake of Amino Acids from Wheat Straw Mimics for Both MIL-140C and DOWEX® 50Wx2: (i) Full Amino Acid Mixture (pH 4.3), (ii) Only Aromatics Mixture (pH 6.2), (iii) Mixture without Apolar Amino Acids (pH 4.3), and (iv) Mixture without Acidic Amino Acids (pH 7.6) ${ }^{a}$

\begin{tabular}{|c|c|c|c|c|c|c|c|c|c|}
\hline \multirow[b]{2}{*}{ amino acid } & \multirow[b]{2}{*}{ concentration $(\mathrm{mM})$} & \multicolumn{4}{|c|}{ uptake on MIL-140C (wt \%) } & \multicolumn{4}{|c|}{ uptake on DOWEX 50Wx2 (wt \%) } \\
\hline & & $\operatorname{mix} 1$ & $\operatorname{mix} 2$ & $\operatorname{mix} 3$ & $\operatorname{mix} 4$ & $\operatorname{mix} 1$ & $\operatorname{mix} 2$ & $\operatorname{mix} 3$ & $\operatorname{mix} 4$ \\
\hline \multicolumn{10}{|c|}{ apolar, aromatic side chain } \\
\hline L-Tyr & 2.3 & 0.21 & 1.1 & 0.21 & 0.56 & 0.62 & 11.5 & 0.9 & 1.15 \\
\hline L-Trp & 5 & 2.4 & 6.0 & 3.1 & 3.7 & 7.7 & 31.6 & 11.1 & 8.67 \\
\hline L-Phe & 4 & 0.44 & 2.2 & 0.58 & 1.2 & 0.63 & 21.4 & 1.2 & 1.72 \\
\hline \multicolumn{10}{|c|}{ apolar, aliphatic side chain } \\
\hline L-Ala & 15 & nu & & & 0.40 & nu & & & 1.2 \\
\hline L-Val & 14 & $<0.10$ & & & 0.87 & nu & & & 1.9 \\
\hline L-Leu & 10 & 0.59 & & & 1.3 & $\mathrm{nu}$ & & & 2.0 \\
\hline L-Ile & 5 & 0.15 & & & 0.62 & nu & & & 0.90 \\
\hline L-Met & 3 & 0.19 & & & 0.72 & nu & & & 0.61 \\
\hline \multicolumn{10}{|c|}{ polar, acidic side chain } \\
\hline L-Asp & 17 & 4.0 & & 4.8 & & nu & & nu & \\
\hline L-Glu & 18 & 3.4 & & 4.2 & & nu & & nu & \\
\hline \multicolumn{10}{|c|}{ polar, alkaline side chain } \\
\hline L-Lys & 7 & $\mathrm{nu}$ & & $\mathrm{nu}$ & 1.5 & 14.5 & & 22.7 & 18 \\
\hline L-Arg & 6 & $\mathrm{nu}$ & & nu & 2.7 & 17.7 & & 22.1 & 16.1 \\
\hline $\mathrm{L}-\mathrm{His}$ & 2.5 & nu & & nu & 0.52 & 5.1 & & 8.3 & 9.2 \\
\hline \multicolumn{10}{|c|}{ polar, uncharged side chain and others } \\
\hline L-Gly & 15 & $<0.10$ & & $<0.10$ & 0.16 & nu & & nu & 1.2 \\
\hline L-Thr & 9 & $<0.10$ & & $\mathrm{nu}$ & 0.72 & nu & & $\mathrm{nu}$ & 0.43 \\
\hline L-Ser & 13 & $<0.10$ & & $<0.10$ & 0.86 & $\mathrm{nu}$ & & $\mathrm{nu}$ & 0.74 \\
\hline L-Pro & 18 & $<0.10$ & & $<0.10$ & $<0.10$ & $<0.10$ & & $<0.10$ & 1.51 \\
\hline L-Cys & 7 & 0.98 & & & $\mathrm{nu}$ & 1.9 & & & $\mathrm{nu}$ \\
\hline
\end{tabular}

${ }^{a}$ Mixture 1: all amino acids, except for L-Gln and L-Asn ( $\mathrm{pH}$ 4.3). Mixture 2: aromatic amino acids (L-Phe, L-Tyr, and L-Trp) (pH 6.2). Mixture 3: all amino acids, except for L-Gln, L-Asn, and amino acids with an apolar side-chain (L-Ala, L-Val, L-Leu, L-Ile, L-Cys, and L-Met) (pH 4.3). Mixture 4: all amino acids, except for L-Gln, L-Asn, and their acidic counterparts L-Glu and L-Asp (pH 7.6). nu: no uptake.

(hetero)aromatic amino acids (L-Tyr, L-Phe, L-Trp, and L-His) display large uptakes. As expected for a material with a permanent negative charge, the acidic amino acids were rejected, while apolar, aliphatic amino acids coadsorb only weakly on the DOWEX 50Wx2 resin. The order of amino acid uptake on DOWEX 50Wx2 is hence largely determined by the properties of the side chain: positively charged, basic $>$ apolar, aromatic > apolar, aliphatic > polar, with no uptake of negatively charged, acidic amino acids at all.

For MIL-140C, besides the expected uptake of L-Trp, L-Phe, and L-Tyr, a clear coadsorption of L-Asp and L-Glu was observed near $\mathrm{pH} 4.3$ (see Table 2). The adsorption of L-Asp and $\mathrm{L}-\mathrm{Glu}$ has also been observed previously in isoreticular $\mathrm{Al}^{3+}$ based metal-organic frameworks. ${ }^{48}$ Uptake of L-Glu on MIL$140 \mathrm{C}$ from single-compound solutions at natural $\mathrm{pH}$ can reach up to 11 wt \% (Figure S10). To evaluate the effect of L-Asp and L-Glu on the adsorption of the AAAs, the mechanism of adsorption of the acidic amino acids needs to be understood. The natural $\mathrm{pH}$ of $\mathrm{L}-\mathrm{Glu}$ solutions is in the range $3.2-3.8$ due to partial proton loss from the side chain's carboxylic group. For very dilute samples $(0.8 \mathrm{mM}, \mathrm{pH} 3.8)$, more than $90 \%$ of glutamic acid was adsorbed on MIL-140C (Figure S10) with full structure retention (Figure S11). This process involved a $\mathrm{pH}$ shift to 6.4 after adsorption, indicating that L-Glu adsorbs in a neutral form on the MIL-140C. This implies that the carboxylate is reprotonated upon adsorption, causing the observed $\mathrm{pH}$ increase in the liquid phase. The presence of a protonated $-\mathrm{C}(=\mathrm{O})-\mathrm{O}-\mathrm{H}$ moiety in the adsorbed $\mathrm{L}-\mathrm{Glu}$ also suggests that a similar hydrogen-bonding with the $\mathrm{Zr}$ - carboxylate chains may be operative as proposed before for LTrp with its indole $-\mathrm{NH}$ moiety.

When MIL-140C is exposed to a complex amino acid mixture from which L-Glu and L-Asp have been omitted (at $\mathrm{pH}$ 7.6), mainly apolar compounds (L-Ile, L-Leu) and L-Arg coadsorb to a fair extent on MIL-140C, together with the AAAs (Table 2). The overall adsorption preference on MIL140C therefore appears to be the following: negatively charged, acidic $>$ aromatic $>$ positively charged, basic $>$ apolar $>$ polar amino acids. These first experiments offer appealing avenues for the use of MOFs, specifically MIL-140C, for the separation of essential aromatic amino acids L-Phe, L-Tyr, and L-Trp from protein hydrolysates in batch and continuous adsorption operation.

\section{CONCLUSIONS}

In this work, we demonstrated that some water-stable MOFs, and especially MIL-140C, can be readily applied as adsorbent media for removing aromatic amino acids from aqueous streams, rivalling ion-exchange resins and zeolites. Uptakes of $20 \mathrm{wt} \%$ for L-Phe and 15 wt \% for L-Trp were achieved. The reusability of MIL-140C was demonstrated for three cycles of batch adsorption and desorption of L-Phe and L-Trp with full retention of adsorption capacity and crystallinity. Aqueous ethanol was sufficient to disrupt the weak dispersive $\pi-\pi$ and hydrogen bonding interactions between the adsorbate and the framework, which is a much milder treatment compared to the regeneration of ion-exchangers under highly acidic or alkaline conditions. 
MIL-140C exhibited an affinity order L-Trp $\gg$ L-Tyr $\geq$ L-Phe when only aromatic amino acids are considered. This affinity order was rationalized by investigating the splitting of the $\nu \mathrm{N}-$ $\mathrm{H}$ vibration of the indole moiety in L-Trp due to hydrogen bonding mechanisms with the double $\mathrm{Zr}$-oxide chain in the MIL-140C framework. The affinity order between L-Phe and LTrp was preserved in a continuous column adsorption experiment. These results indicate that MIL-140C has the potential to recover aromatic amino acids from mixtures for analytical AAA separation but also for workup of singlecompound fermentation broths, using this material as active separation adsorbent in columns.

Studies with model mixtures that mimic the amino acid composition of a wheat straw protein hydrolysate showed a fair uptake of aromatic amino acids with considerable coadsorption of mainly amino acids with acidic aliphatic side chains. In light of these results, we emphasize the promising potential to apply MOFs as adsorbents in amino acid separations and recovery.

\section{ASSOCIATED CONTENT}

\section{S Supporting Information}

The Supporting Information is available free of charge on the ACS Publications website at DOI: 10.1021/acsami.7b09175.

Experimental details and additional PXRD spectra, aromatic amino acid adsorption isotherms, full FT-IR spectra of MIL-140C with adsorbed L-Trp and L-Phe and after washing, exemplary HPLC chromatograms, SEM micrographs, $\mathrm{N}_{2}$-physisorption isotherm, initial concentrations for the wheat straw protein hydrolysate model mixtures, and an additional note on the FT-IR analysis (PDF)

\section{AUTHOR INFORMATION}

\section{Corresponding Author}

*E-mail: dirk.devos@kuleuven.be.

ORCID 1

Dries Jonckheere: 0000-0002-1192-5004

Julian A. Steele: 0000-0001-7982-4413

Birgit Claes: 0000-0002-8851-0739

Maarten B. J. Roeffaers: 0000-0001-6582-6514

Notes

The authors declare no competing financial interest.

\section{ACKNOWLEDGMENTS}

D.E.D.V. acknowledges the Research Foundation Flanders (FWO Vlaanderen) (Project G.0256.14N) for support in the frame of research projects, the Flemish government (Methusalem CASAS2), and the Belgian Science Policy Office Interuniversity Attraction Poles (IAP) program in the frame of IAP 7/05 for financial support. Further, we are grateful to Flanders Innovation \& Entrepreneurship (L.C.; Grant HBC.2016.0242) and to the Research Foundation Flanders (FWO Vlaanderen) (B.B.; Grant 12R1217N) for individual postdoctoral fellowships.

\section{ABBREVIATIONS}

AA, amino acid

AAA, aromatic amino acid

FT-IR, Fourier transform infrared

fwhm, full width at half-maximum

HPLC, high pressure liquid chromatography

\author{
L-Ala, L-alanine \\ L-Asn, L-asparagine \\ L-Arg, L-arginine \\ L-Asp, L-aspartic acid \\ L-Cys, L-cysteine \\ L-Gln, L-glutamine \\ L-Glu, L-glutamic acid \\ L-Gly, L-glycine \\ L-His, L-histidine \\ L-Ile, L-isoleucine \\ L-Leu, L-leucine \\ L-Lys, L-lysine \\ L-Met, L-methionine \\ L-Phe, L-phenylalanine \\ L-Pro, L-proline \\ L-Ser, L-serine \\ L-Thr, L-threonine \\ L-Trp, L-tryptophan \\ L-Tyr, L-tyrosine \\ L-Val, L-valine \\ MIL, Matériaux de l'Institut Lavoisier \\ MOF, metal-organic framework \\ PS/DVB, polystyrene cross-linked with divinylbenzene \\ PXRD, powder X-ray diffraction \\ TFA, trifluoroacetic acid
}

\section{REFERENCES}

(1) Leuchtenberger, W.; Huthmacher, K.; Drauz, K. Biotechnological Production of Amino Acids and Derivatives: Current Status and Prospects. Appl. Microbiol. Biotechnol. 2005, 69, 1-8.

(2) Daubner, S. C.; Le, T.; Wang, S. Tyrosine Hydroxylase and Regulation of Dopamine Synthesis. Arch. Biochem. Biophys. 2011, 508, $1-12$.

(3) Fukuwatari, T.; Shibata, K. Nutritional Aspect of Tryptophan Metabolism. Int. J. Tryptophan Res. 2013, 6, 3-8.

(4) Scott, E.; Peter, F.; Sanders, J. Biomass in the Manufacture of Industrial Products - The Use of Proteins and Amino Acids. Appl. Microbiol. Biotechnol. 2007, 75, 751-762.

(5) Sanders, J.; Scott, E.; Weusthuis, R.; Mooibroek, H. Bio-Refinery as the Bio-Inspired Process to Bulk Chemicals. Macromol. Biosci. 2007, $7,105-117$

(6) Tuck, C. O.; Pérez, E.; Horváth, I. T.; Sheldon, R. A.; Poliakoff, M. Valorization of Biomass: Deriving More Value from Waste. Science 2012, 337, 695-699.

(7) Demain, A. L. REVIEWS: The Business of Biotechnology. Ind. Biotechnol. 2007, 3, 269-283.

(8) RadientInsights Amino Acids Market Size \& Research Report, 2022; 2015. http://www.radiantinsights.com/research/amino-acidsmarket (accessed June 15, 2017); p 273.

(9) Global Industry Analysts Inc. Healthy Market for Meat and Nutraceuticals Drive the Global Amino Acids Market, 2017. http:// www.strategyr.com/MarketResearch/Amino_Acids_Market_Trends. asp (accessed June 15, 2017).

(10) Ikeda, M. Amino Acid Production Processes. In Microbial Production of L-Amino Acids; Faurie, R., Thommel, J., Bathe, B., Debabov, V. G., Huebner, S., Ikeda, M., Kimura, E., Marx, A., Möckel, B., Mueller, U., Pfefferle, W., Eds.; Springer: Berlin, Heidelberg, 2003; pp 1-35.

(11) Sugimoto, M.; Flickinger, M. C. Amino Acids, Production Processes. In Encyclopedia of Industrial Biotechnology: Bioprocess, Bioseparation, and Cell Technology; John Wiley \& Sons, Inc.: Hoboken, NJ, 2009.

(12) Lammens, T. M.; Franssen, M. C. R.; Scott, E. L.; Sanders, J. P. M. Availability of Protein-Derived Amino Acids as Feedstock for the Production of Bio-Based Chemicals. Biomass Bioenergy 2012, 44, 168181. 
(13) Kattan Readi, O. M.; Gironès, M.; Nijmeijer, K. Separation of Complex Mixtures of Amino Acids for Biorefinery Applications using Electrodialysis. J. Membr. Sci. 2013, 429, 338-348.

(14) Takors, R. Model-Based Analysis and Optimization of an ISPR Approach using Reactive Extraction for Pilot-Scale L-Phenylalanine Production. Biotechnol. Prog. 2004, 20, 57-64.

(15) Bi, P. Y.; Dong, H. R.; Yu, H. B.; Chang, L. A New Technique for Separation and Purification of L-Phenylalanine from Fermentation Liquid: Flotation Complexation Extraction. Sep. Purif. Technol. 2008, 63, 487-491.

(16) Hong, S. U.; Bruening, M. L. Separation of Amino Acid Mixtures using Multilayer Polyelectrolyte Nanofiltration Membranes. J. Membr. Sci. 2006, 280, 1-5.

(17) Capela, E. V.; Quental, M. V.; Domingues, P.; Coutinho, J. A. P.; Freire, M. G. Effective Separation of Aromatic and Aliphatic Amino Acid Mixtures using Ionic-Liquid-Based Aqueous Biphasic Systems. Green Chem. 2017, 19, 1850-1854.

(18) Nozaki, S.; Murata, N.; Miyazaki, K. Separation Method of Amino Acids. Google Patents US 5300653 A, 1994.

(19) Luo, W.; Chen, H.; Fan, L.; Huang, J.; Huang, L.; Xu, Z.; Cen, P. Adsorption Behavior of L-Tryptophan on Ion-Exchange Resin. Korean J. Chem. Eng. 2011, 28, 1272-1279.

(20) Luo, W.; Wei, P.; Chen, H.; Fan, L.; Huang, L.; Huang, J.; Xu, Z.; Cen, P. Kinetics and Optimization of L-Tryptophan Separation With Ion-Exchange Chromatography. Korean J. Chem. Eng. 2011, 28, $1280-1285$.

(21) Itoh, H.; Toide, K.; Ikeda, M. Method for Purification of an Amino Acid using Ion Exchange Resin. Google Patents US 5279744 A, 1994.

(22) Yonsel, S.; Schafer-Treffenfeldt, W.; Kiss, A.; Sextl, E.; Kinz, H. Process for the Separation of Amino Acids from Aqueous Solutions. Google Patents US 5312980 A, 1994.

(23) Munsch, S.; Hartmann, M.; Ernst, S. Adsorption and Separation of Amino Acids from Aqueous Solutions on Zeolites. Chem. Commun. 2001, 1978-1979.

(24) Titus, E.; Kalkar, A. K.; Gaikar, V. G. Equilibrium Studies of Adsorption of Amino Acids on NaZSM-5 Zeolite. Colloids Surf., A 2003, 223, 55-61.

(25) Krohn, J. E.; Tsapatsis, M. Amino Acid Adsorption on Zeolite $\beta$. Langmuir 2005, 21, 8743-8750.

(26) Krohn, J. E.; Tsapatsis, M. Phenylalanine and Arginine Adsorption in Zeolites X, Y, and $\beta$. Langmuir 2006, 22, 9350-9356.

(27) Carneiro, C. E. A.; de Santana, H.; Casado, C.; Coronas, J.; Zaia, D. A. M. Adsorption of Amino Acids (Ala, Cys, His, Met) on Zeolites: Fourier Transform Infrared and Raman Spectroscopy Investigations. Astrobiology 2011, 11, 409-418.

(28) Stückenschneider, K.; Merz, J.; Hanke, F.; Rozyczko, P.; Milman, V.; Schembecker, G. Amino-Acid Adsorption in MFI-Type Zeolites Enabled by the pH-Dependent Ability to Displace Water. J. Phys. Chem. C 2013, 117, 18927-18935.

(29) Stückenschneider, K.; Merz, J.; Schembecker, G. Molecular Interaction of Amino Acids with Acidic Zeolite BEA: The Effect of Water. J. Phys. Chem. C 2014, 118, 5810-5819.

(30) O'Connor, A. J.; Hokura, A.; Kisler, J. M.; Shimazu, S.; Stevens, G. W.; Komatsu, Y. Amino Acid Adsorption onto Mesoporous Silica Molecular Sieves. Sep. Purif. Technol. 2006, 48, 197-201.

(31) Gao, Q.; Xu, W.; Xu, Y.; Wu, D.; Sun, Y.; Deng, F.; Shen, W. Amino Acid Adsorption on Mesoporous Materials: Influence of Types of Amino Acids, Modification of Mesoporous Materials, and Solution Conditions. J. Phys. Chem. B 2008, 112, 2261-2267.

(32) Vinu, A.; Hossain, K. Z.; Kumar, G. S.; Sivamurugan, V.; Ariga, K. Adsorption of Amino Acid on Mesoporous Molecular Sieves. Stud. Surf. Sci. Catal. 2005, 156, 631-636.

(33) Janiak, C. Engineering Coordination Polymers towards Applications. Dalton Trans. 2003, 2781-2804.

(34) Ferey, G. Hybrid Porous Solids: Past, Present, Future. Chem. Soc. Rev. 2008, 37, 191-214.
(35) Janiak, C.; Vieth, J. K. MOFs, MILs and More: Concepts, Properties and Applications for Porous Coordination Networks (PCNs). New J. Chem. 2010, 34, 2366-2388.

(36) Li, B.; Wang, H.; Chen, B. Microporous Metal-Organic Frameworks for Gas Separation. Chem. - Asian J. 2014, 9, 1474-1498.

(37) Corma, A.; Garcia, H.; Xamena, F. X. L. Engineering Metal Organic Frameworks for Heterogeneous Catalysis. Chem. Rev. 2010, $110,4606-4655$

(38) Valvekens, P.; Vermoortele, F.; De Vos, D. Metal-Organic Frameworks as Catalysts: The Role of Metal Active Sites. Catal. Sci. Technol. 2013, 3, 1435-1445.

(39) Perry, J. J.; Bauer, C. A.; Allendorf, M. D. Luminescent MetalOrganic Frameworks. In Metal-Organic Frameworks; Wiley-VCH Verlag GmbH \& Co. KGaA: Hoboken, NJ, 2011; pp 267-308.

(40) Cui, Y.; Yue, Y.; Qian, G.; Chen, B. Luminescent Functional Metal-Organic Frameworks. Chem. Rev. 2012, 112, 1126-1162.

(41) Hu, Z.; Deibert, B. J.; Li, J. Luminescent Metal-Organic Frameworks for Chemical Sensing and Explosive Detection. Chem. Soc. Rev. 2014, 43, 5815-5840.

(42) Chowdhury, M. A. The Applications of Metal-Organic Frameworks in Controlled Release of Drugs. Rev. J. Chem. 2017, 7, $1-22$.

(43) Van de Voorde, B.; Bueken, B.; Denayer, J.; De Vos, D. Adsorptive Separation on Metal-Organic Frameworks in the Liquid Phase. Chem. Soc. Rev. 2014, 43, 5766-5788.

(44) Marshall, R. J.; Hobday, C. L.; Murphie, C. F.; Griffin, S. L.; Morrison, C. A.; Moggach, S. A.; Forgan, R. S. Amino Acids as Highly Efficient Modulators for Single Crystals of Zirconium and Hafnium Metal-Organic Frameworks. J. Mater. Chem. A 2016, 4, 6955-6963.

(45) Gutov, O. V.; Molina, S.; Escudero-Adán, E. C.; Shafir, A. Modulation by Amino Acids: Toward Superior Control in the Synthesis of Zirconium Metal-Organic Frameworks. Chem. - Eur. J. 2016, 22, 13582-13587.

(46) Liang, K.; Ricco, R.; Doherty, C. M.; Styles, M. J.; Falcaro, P. Amino Acids as Biomimetic Crystallization Agents for the Synthesis of ZIF-8 Particles. CrystEngComm 2016, 18, 4264-4267.

(47) De, D.; Pal, T. K.; Bharadwaj, P. K. A Porous Cu(II)-MOF with Proline Embellished Cavity: Cooperative Catalysis for the BaylisHillman Reaction. Inorg. Chem. 2016, 55, 6842-6844.

(48) Nakahama, M.; Reboul, J.; Yoshida, K.; Furukawa, S.; Kitagawa, S. L-Glutamic Acid Release from a Series of Aluminum-Based Isoreticular Porous Coordination Polymers. J. Mater. Chem. B 2015, 3, 4205-4212.

(49) Cousin Saint Remi, J.; Rémy, T.; Van Hunskerken, V.; van de Perre, S.; Duerinck, T.; Maes, M.; De Vos, D.; Gobechiya, E.; Kirschhock, C. E. A.; Baron, G. V.; Denayer, J. F. M. Biobutanol Separation with the Metal-Organic Framework ZIF-8. ChemSusChem 2011, 4, 1074-1077.

(50) Lannoeye, J.; Van de Voorde, B.; Bozbiyik, B.; Reinsch, H.; Denayer, J.; De Vos, D. An Aliphatic Copper Metal-Organic Framework as Versatile Shape Selective Adsorbent in Liquid Phase Separations. Microporous Mesoporous Mater. 2016, 226, 292-298.

(51) Van der Helm, D.; Lawson, M. B.; Enwall, E. L. The Crystal Structure of Bis-(L-Phenylalaninato)Copper(II). Acta Crystallogr., Sect. B: Struct. Crystallogr. Cryst. Chem. 1971, 27, 2411-2418.

(52) Xie, Y.; Wu, H.-H.; Yong, G.-P.; Wang, Z.-Y. rac-Poly[bis([mu]Tryptophanato)Manganese(II)]. Acta Crystallogr., Sect. E: Struct. Rep. Online 2006, 62, m2089-m2090.

(53) Wang, J.; Xu, X.; Ma, W.; Lu, L.; Yang, X. rac-catenaPoly[nickel(II)-di-[mu]-tryptophanato]. Acta Crystallogr., Sect. E: Struct. Rep. Online 2007, 63, m2867-m2868.

(54) Van de Voorde, B.; Damasceno Borges, D.; Vermoortele, F.; Wouters, R.; Bozbiyik, B.; Denayer, J.; Taulelle, F.; Martineau, C.; Serre, C.; Maurin, G.; De Vos, D. Isolation of Renewable Phenolics by Adsorption on Ultrastable Hydrophobic MIL-140 Metal-Organic Frameworks. ChemSusChem 2015, 8, 3159-3166.

(55) Miura, T.; Takeuchi, H.; Harada, I. Characterization of Individual Tryptophan Side Chains in Proteins Using Raman 
Spectroscopy and Hydrogen-Deuterium Exchange Kinetics. Biochemistry 1988, 27, 88-94.

(56) Joseph, J.; Jemmis, E. D. Red-, Blue-, or No-Shift in Hydrogen Bonds: A Unified Explanation. J. Am. Chem. Soc. 2007, 129, 46204632.

(57) Kumar, S.; Das, A. Mimicking Trimeric Interactions in the Aromatic Side Chains of the Proteins: a Gas Phase Study of Indole... (pyrrole) ${ }_{2}$ Heterotrimer. J. Chem. Phys. 2012, 136, 174302-174302.

(58) Kumar, S.; Das, A. Observation of Exclusively $\pi$-Stacked Heterodimer of Indole and Hexafluorobenzene in the Gas Phase. J. Chem. Phys. 2013, 139, 104311-104311.

(59) Li, X.; Liu, L.; Schlegel, H. B. On the Physical Origin of BlueShifted Hydrogen Bonds. J. Am. Chem. Soc. 2002, 124, 9639-9647.

(60) Yang, Y.; Zhang, W.; Gao, X. Blue-Shifted and Red-Shifted


Complexes. Int. J. Quantum Chem. 2006, 106, 1199-1207.

(61) Liu, Y.; Liu, W.; Li, H.; Yang, Y.; Cheng, S. Interaction of the Important Species HNO and HFSO2 in the Atmosphere: Theoretical Study of the N-H and S-H Blue-Shifted Hydrogen Bonds. Int. J. Quantum Chem. 2007, 107, 396-402.

(62) Solimannejad, M.; Massahi, S.; Alkorta, I. A Computational Study of Dimers and Trimers of Nitrosyl Hydride: Blue Shift of NH Bonds That Are Involved in H-Bond and Orthogonal Interactions. Chem. Phys. 2009, 362, 1-7.

(63) Solimannejad, M.; Nassirinia, N.; Amani, S. HNO(H2O) n ( $\mathrm{n}=$ 1-4) Clusters: A Theoretical Study. Struct. Chem. 2011, 22, 865-871.

(64) Liu, Y.; Liu, W.; Li, H.; Liu, J.; Yang, Y. Theoretical Study of Hydrogen Bonding Interaction in Nitroxyl (HNO) Dimer: Interrelationship of the Two $\mathrm{N}-\mathrm{H} \cdots \mathrm{O}$ Blue-Shifting Hydrogen Bonds. J. Phys. Chem. A 2006, 110, 11760-11764.

(65) Van de Voorde, B.; Stassen, I.; Bueken, B.; Vermoortele, F.; De Vos, D.; Ameloot, R; Tan, J.-C.; Bennett, T. D. Improving the Mechanical Stability of Zirconium-Based Metal-Organic Frameworks by Incorporation of Acidic Modulators. J. Mater. Chem. A 2015, 3, $1737-1742$.

(66) Ryder, M. R.; Civalleri, B.; Tan, J.-C. Isoreticular ZirconiumBased Metal-Organic Frameworks: Discovering Mechanical Trends and Elastic Anomalies Controlling Chemical Structure Stability. Phys. Chem. Chem. Phys. 2016, 18, 9079-9087. 